\title{
Carbon Nanofiber Aggregate Sensors for Sustaining Resilience of Civil Infrastructures to Multi-Hazards
}

\author{
Avinash Gautam ${ }^{1}$, Mo $\mathrm{YL}^{1 *}$, Yuhua Chen ${ }^{2}$, Jinghong Chen ${ }^{2}$ and Bhagirath Joshi ${ }^{1}$ \\ ${ }^{1}$ Department of Civil and Environmental Engineering, USA \\ ${ }^{2}$ Department Electrical and Computer Engineering, USA \\ *Corresponding author: Mo YL, Department of Civil and Environmental Engineering, USA \\ Submission: 海 January 16, 2019; Published: 海 : February 06, 2019
}

\begin{abstract}
Natural disasters (earthquakes, hurricanes, tornadoes. and floods) or man-made disasters or accidents (missile/aircraft impacts, fires. and explosions) lead to substantial damage on critical infrastructures and communities and have social, economic, and environmental consequences. As the safety of civil infrastructures is critically important, effective structural health monitoring (SHM) systems of infrastructures should be developed to avoid and mitigate the risks caused by various types of hazards. Carbon Nanofiber Aggregates (CNFAs) developed at the University of Houston are piezoelectric aggregates that can respond uniquely to different multi-hazards. This unique response makes this a sensor capable of being used in the Structural Health Monitoring (SHM) of civil infrastructures in each of the multi-hazards. This paper explains how CNFAs can be used for multi-hazard monitoring and discusses the ultra-low noise, high sensitivity of CNFA sensor electrical interfaces, radiation-tolerant wired and wireless communication capabilities of the CNFA sensor which leads to the very possible development of the wireless CNFAs' real-time SHM system for sustaining resilience of civil infrastructures to multi-hazards.
\end{abstract}

\section{Introduction}

The safety of civil infrastructures in operation and maintenance is closely related to the social, economic, and environmental benefits and is always drawing the attention of the engineering field and academia. However, the loading condition of these infrastructures is not consistent during the service period. The scientific design should also include the influence of natural disasters (earthquakes, hurricanes, tornadoes, and floods) and human-made disasters or accidents (missile/aircraft impacts, fires, and explosions) which lead to substantial damage on critical infrastructures and affect social, economic, and environmental aspects. As the safety of infrastructures is critically important, effective structural health monitoring (SHM) systems of civil infrastructures should be developed to avoid and mitigate the risks caused by various types of hazards.

This paper explains the development of a reliable and costeffective wired carbon nanofiber aggregate (CNFA) sensor that can be deployed to surrounding civil infrastructures for achieving realtime structural health monitoring on multi-hazards with wireless technology characterized with signal transmission and acquisition techniques, as presented in Figure 1. The stress level under the effect of earthquakes, tornados, flooding, and the impact of missile/ aircraft attacks and explosions is effectively detected by the CNFA strain sensing capability. The depth of water accumulated due to flooding is determined by taking into account the total hydrostatic pressure applied on the surface of the CNFA. This hydrostatic pressure or structural stress produces equivalent stress in the CNFA which is derived from the strain generated in it. The CNFA based multi-hazards monitoring system is employed to determine the flood water depth and the real-time stress level of civil infrastructures subjected to natural and human-made disasters and wirelessly transmit the information to a central monitoring system to immediately guide operators and to provide accurate stress measures for post-disaster damage assessment.

Thus, this paper's major objectives are as follows:

A. Investigate the application of the novel CNFA based multihazard monitoring system to civil infrastructures for sustaining resilience.

B. Develop ultra-low noise and high sensitivity CNFA sensor electrical interfaces for accurate structural stress measurement.

C. Develop radiation-tolerant wired and wireless communication capabilities of CNFA sensors for reliable operations near civil infrastructures.

D. Employ the proposed real-time SHM system for civil infrastructures subjected to multi-hazards using wired and study the possibility of wireless CNFA sensors.

E. To ensure the stability and dependability of the proposed CNFA-based SHM system, the conventional wired-dataacquisition approach is adopted in the practical application in civil 
infrastructures. Moreover, the wireless data collection technique is developed to further equip the research with the advanced and latest science and technology related to this topic.

\section{Methodology}

The paper is based on novel CNFAs, small sensing cubes made of mortar with additives including carbon nanofibers (CNFs), developed at the University of Houston. CNFs are graphene ringbased materials with aspect ratios greater than 1000 and are characterized by high surface areas. The electrical conductivity of the fibers enables the DC electrical resistivity of the composites to change in response to strain or temperature, allowing sensing [1-7]. CNF concrete, which is classified as a smart material, exhibits properties necessary for reversible strain monitoring and electromagnetic interference (EMI) shielding due to the tunnel conductivity effect [6]. The development of CNFA sensors has played a vital role in multi-hazard SHM since the strain sensing capabilities of the CNFAs reveal the stress that the civil infrastructures experience during a natural or human-made disaster and facilitate post-disaster damage assessment of the civil infrastructures even without reconnaissance.

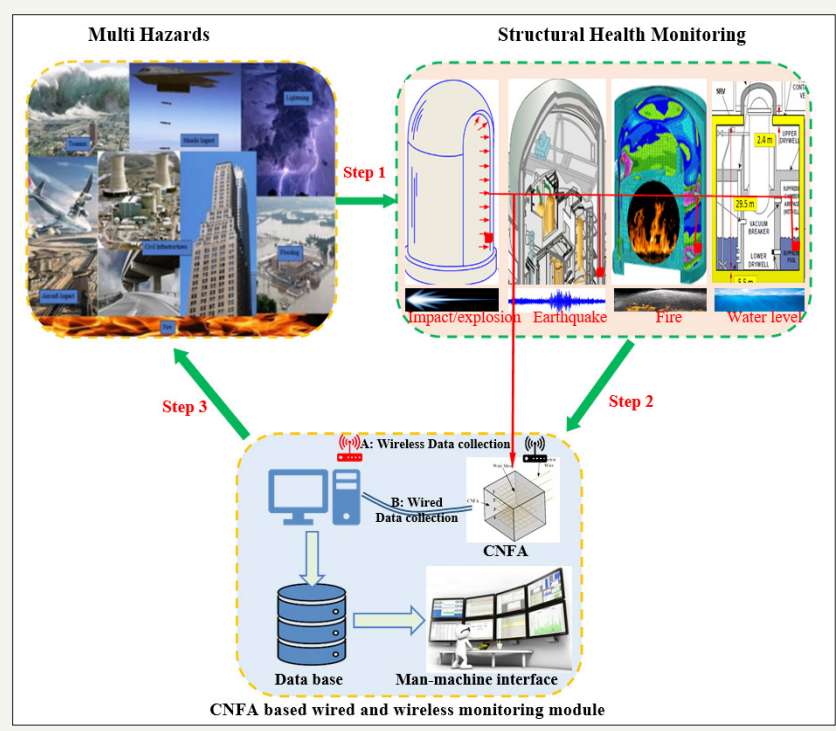

Figure 1: Flowchart for a real-time structural monitoring system for multi-hazards on civil infrastructures using wired and wireless CNFA sensors.

The concept of strain sensing refers to the ability to measure an electrical or optical response corresponding to a strain. Thus, the electrical resistance can be determined in the CNFAs through the embedment of four metal meshes and the use of the fourprobe method. The relationship between the electrical resistance variation (ERV) and the observed strain for plain self-consolidating concrete (SCC) and SCC cylinders containing the CNF, PR-19-XTLHT-OX, ranging from $0.01 \%$ to $0.06 \%$ by the weight of cement, when subjected to compression are shown in Figure 2. It can be seen from the figure that the resistance decreases for all concentrations of CNFs. The concentration of CNFs in concrete plays a crucial role in achieving the optimum performance where the concentration will differ depending upon the type of the CNFs preferred for the application [6]. More specifically, the relationship between the ERV and the stress can be determined by the equations presented in Figure 3. Similarly, Figure 4 indicates the ERV and water level relationship. The dotted line expresses the experimental data set while the solid line shows the curve fitted by the proposed equations which are quite identical.

Since the optimum concentration of well-dispersed CNFs allows for the greatest electrical resistance variation of concrete with increasing strain, CNFAs can be used in applications that require strain monitoring. This principle is used to evaluate the stress produced in CNFA sensors in civil infrastructures subjected to earthquakes, tornados, and missile impacts, etc. Also, due to the hydrostatic pressure developed as a result of water accumulated in the lower regions of civil infrastructures during flooding, the CNFA sensors are successfully used to determine the flood water level.

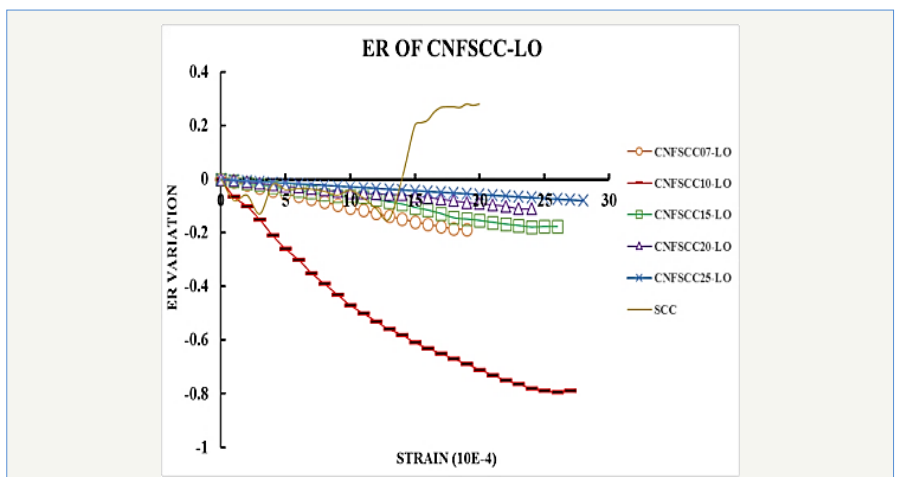

Figure 2: ERV for all concentrations of CNFSCC-LO [6].

\section{Development of Carbon Nano Fiber Aggregates (CNFAs)}

As discussed above, the University of Houston [1] discovered that concrete material is conductive when CNFs are added to the 
concrete mix. This discovery is very significant because CNF concrete itself is a kind of strain sensor, i.e., it has a self-sensing capability and hence the developed sensor will have the same life-span as that of the concrete infrastructure. Nevertheless, a systematic study on the development of CNFAs was not performed. To apply this discovered material science and engineering to civil infrastructures, CNFAs were developed in this research by conducting a thorough and rigorous investigation to sustain resilience of civil infrastructures to multi-hazards.

The CNFA is typically a one-inch cube $(2.54 \mathrm{~cm} \times 2.54 \mathrm{~cm} \times 2.54 \mathrm{~cm})$ of mortar which contains a certain percentage of CNFs by the weight of cement. The electrical resistance was measured in the CNFAs through the embedment of four metal meshes and the use of the four-probe method. Figure 5 represents a schematic showing the CNFA with the four embedded meshes and Figure 5 demonstrates the arrangement of the four-probe method. In this method, the current is supplied to a pair of current leads (1 and 4) and the voltage drop can be measured across the inner connections (2 and 3). The electrical resistance $\mathrm{Ri}$ and electrical resistance variation $\Delta \mathrm{Ri}$ was calculated using $\mathrm{Ri}=\mathrm{Vi} / \mathrm{Ai}$ and $\Delta \mathrm{Ri}=(\mathrm{Ri}-\mathrm{R} 0) /$ R0, respectively, where $\mathrm{Vi}$ and Ai were the recorded values of the voltage and current, respectively, and R0 represented the original electrical resistance.

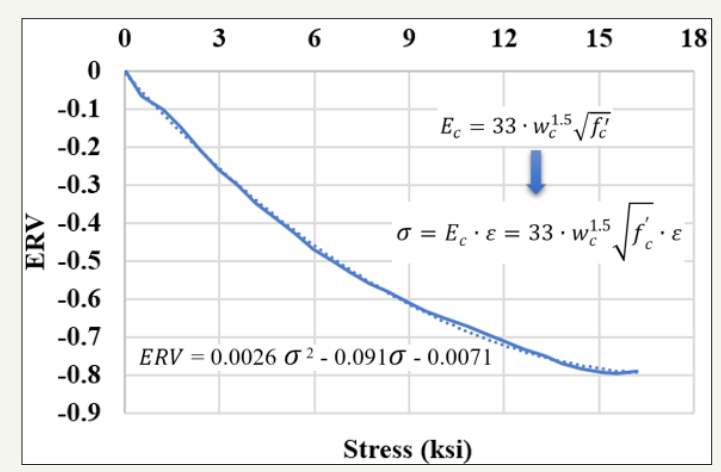

Figure 3: The relationship between ERV and stress.

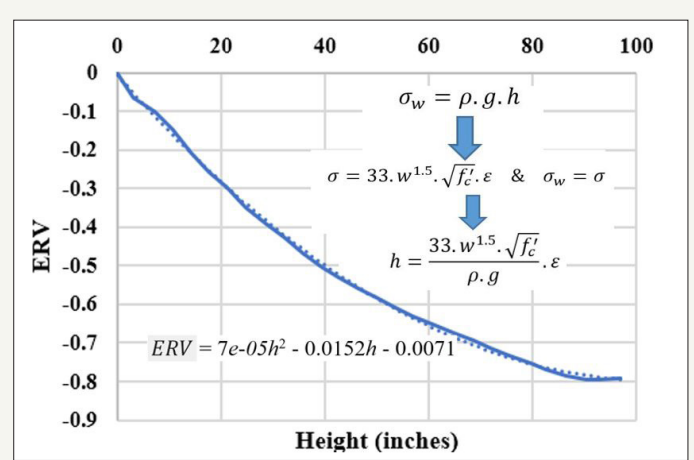

Figure 4: The relationship between ERV and water level.

By utilizing the outcomes of the tests conducted for determining the optimal CNF dosage, a mix design was developed to augment the material and electrical properties. The mixing procedure used for the CNFAs was a hybrid of the mixing procedure proposed for a high-performance self-consolidating steel fiber reinforced concrete mix [8] and the mixing procedure proposed by the University of Houston for a self-consolidating CNF concrete [6]. In this hybrid mixing procedure, the water, high-range water reducer (HRWR), and CNF were premixed and added to the cement, silica fume, and fine aggregates in several steps to create a homogenous paste.

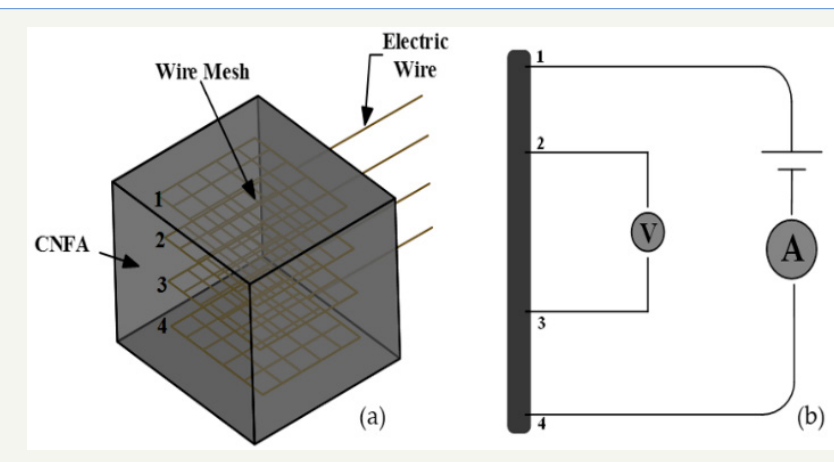

Figure 5: (a) CNFA schematic; (b) the four-probe method.

\section{CNFA size}

For size optimization, the CNFA needed to be designed in such a way that the meshes required for the four-probe method can be easily constructed and accommodated within the aggregate without touching one another. If the CNFA was embedded in concrete, the nominal maximum size of course aggregates should be based on the provisions by the America Concrete Institute (ACI) [9]. Thus, the CNFA size was selected as a one-inch cube $(2.54 \mathrm{~cm}$ by $2.54 \mathrm{~cm}$ by $2.54 \mathrm{~cm}$ ), which takes into consideration the construction limitations as well as manageable space to place the four wire meshes required for the four-probe method.

\section{Mesh construction}
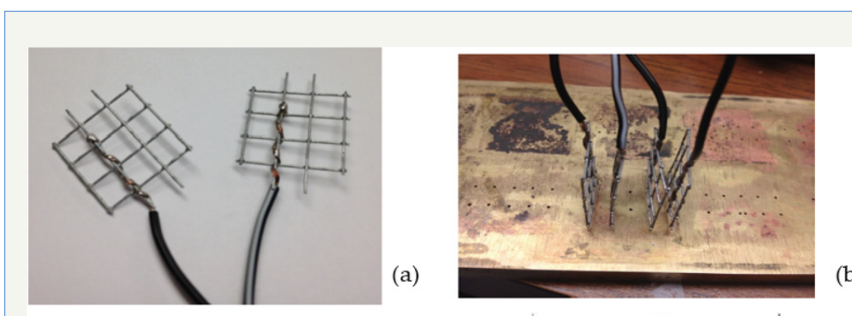

(b)
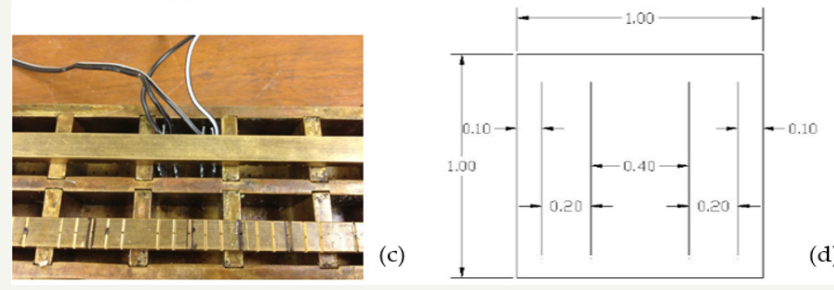

Figure 6: (a) Two meshes with soldered wires; (b) Meshes inserted into the bottom of formwork; (c)Meshes inserted into complete formwork; and (d) Mesh spacing (unit in inch) [1].

The meshes provided in the CNFA were made up of $0.25 \mathrm{in}$. by $0.25 \mathrm{in}$. $(6.35 \mathrm{~mm}$ by $6.35 \mathrm{~mm}) 23$-gauge welded galvanized steel hardware cloth. The hardware cloth was cut into $0.75 \mathrm{in}$. by $0.75 \mathrm{in}$. ( $19.05 \mathrm{~mm}$ by $19.05 \mathrm{~mm}$ ) squares with four prongs extending, as shown in Figure 6. Gauge 24 copper wire was soldered to one of the 
prongs which can be used to reinforce the wire extending from the CNFA. The extended prongs were inserted into holes drilled in the formwork to hold the bottom of the meshes in place during casting, as shown in Figure 6 and a whole arrangement was as shown in Figure 6. The spacing of the meshes is shown in Figure 6.

\section{Mortar mix design}

The CNFA can be made up of a CNF mortar mix. The mortar mix is provided with admixtures along with the typical mortar ingredients such as cement and fine aggregate. The first admixture is a high-range water reducer which assists in creating selfconsolidating concrete (SCC) and benefits in the dispersion of fibers. The mortar must be in the self-consolidating form so that it can flow under its own weight around the meshes without creating any voids with no mechanical vibration. The second admixture that is incorporated into the mix is silica fume. It helps to increase the dispersion of fibers in cement-based materials. The third admixture is the CNFs, which allows the mortar mixture to become self-sensing. The properties of the materials to be used in the mix are as follows:

A. Cement: The cement used is ASTM Type III Portland cement. Type III is chosen for its decreased curing time for quicker CNFA manufacturing.

B. Fine Aggregate: Washed, dried, and screened fine sand.

C. High-Range Water Reducer (HRWR): Glenium ${ }^{\circledR} 3400$ HES is a polycarboxylate admixture.

D. Silica Fume: Rheomac® SF100 is a dry, densified silica fume admixture.

E. Carbon Nanofibers: Pyrograf Products, Inc. PR-19-XTLHT-OX fibers can be tried in the production of CNFA. Reference [6] completed an extensive study on various CNFs and found PR19-XT-LHT-OX fibers to have the best self-sensing performance in concrete.

\section{Radiation-tolerant wireless communication hardware}

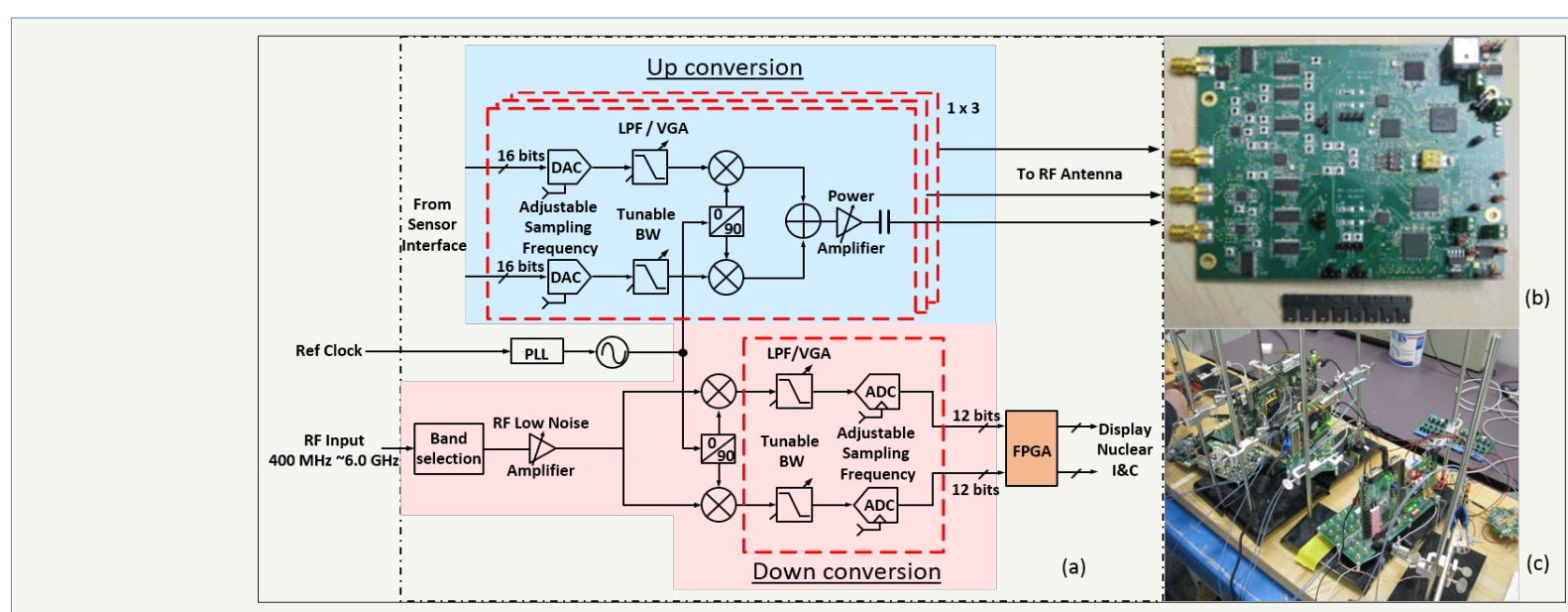

Figure 8: (a) A schematic diagram of the proposed wireless communication board; (b) a wideband RF transceiver hardware design through an NSF funded project [10]; (c) radiation-tolerant PLL testing conducted at LANL [11]. 
As presented in Figure 8, the wireless communication hardware development can leverage our previous R\&D works in which we have demonstrated a wideband cognitive radio transceiver and wireless channel emulator through a National Science Foundation (NSF)-funded project [10], a radiation-hard 6-12 GHz 00.37pS RMS jitter wideband LC-VCO phase-locked loop (PLL) [11], and radiationtolerant application specific integrated circuits (ASICs) for CERN's large hadron collider (LHC) detectors [12-27]. In developing the wireless transceiver, commercial off-the-shelf (COTS) radiationhardened components based on Silicon-on-Sapphire (SOS) and Silicon on Insulator (SOI) technology should be utilized. The SOS and SOI components are inherently more radiation-tolerant in terms of the total ionizing dose (TID) than the complementary metal-oxide-semiconductor (CMOS) technology. Single event effects can also be addressed for logic circuits. Dynamic logic gates can be completely avoided due to their single event update (SEU) sensitivity. The dual interlocked storage cell (DICE) technique can be employed to protect all configuration registers in the system.

\section{Low noise CNFA sensor electrical interface}

To achieve high sensitivity for CNFA strain sensing, low noise high sensitivity sensor interface electronics should be developed. We should start the low noise amplifier design leveraging our previous design experiences for the ATLAS experiments at CERN. The low-noise preamp as shown in Figure 9 is based on the "super common-base" architecture in a Si Ge bipolar process and has proven to offer superior noise performance. To reduce the system cost and facilitate system integration, leveraging our previous experiences on an ultra-low noise pre-amplifier design for high-frequency ultrasound imaging applications [28], we should also investigate the design of the CNFA interface electronics using low-cost CMOS technology. As shown in Figure 9, the proposed CMOS preamp should utilize a novel feedforward noise cancelling strategy, while an auxiliary amplifier generates an out-of-phase noise to cancel the thermal noise of the main amplifier. Figure 9 demonstrates the noise performance with and without noise cancelling.
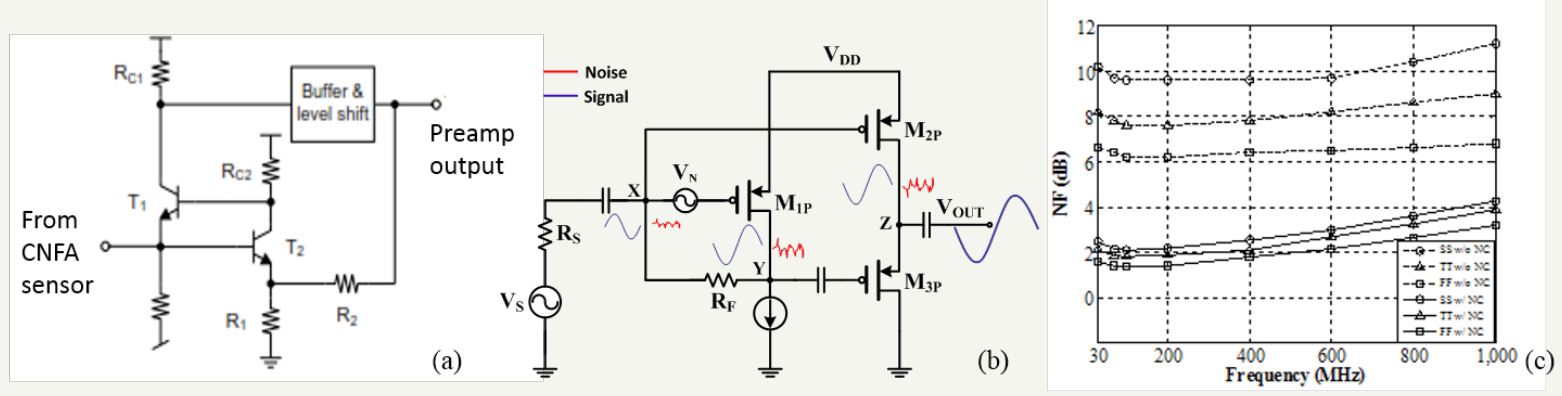

Figure 9: (a) "Super common-base" Si Ge bipolar low-noise preamplifier, (b) CMOS low-noise preamplifier with noise cancelling; and (c) simulated noise performance with and without noise cancelling.

\section{FPGA-based real-time data acquisition and processing}

The hardware implementation of the wireless communication system needs to be capable of real-time signal monitoring and processing with low latency and high-throughput. Field programmable gate arrays (FPGAs) have two key components that are well suited for the proposed application: embedded memories for real-time data acquisition and high-speed embedded multipliers for real-time data processing. Also, the FPGA-based system can be made simpler, more testable and less dependent on complex software, which is the desired feature for civil infrastructure applications. Radiation-tolerant FPGAs or special devices such as those using Silicon on Sapphire or Silicon on Insulator technology should be utilized. Also, radiation-tolerant design techniques should be applied in FPGA hardware implementation.

\section{Electromagnetic interference, cyber-security, reliability, and installation}

The wireless CNFA sensor system should also address critical issues such as electromagnetic and radio frequency interferences (EMI/RFI), cybersecurity, reliability, and installation issues such as the coverage range of the wireless signal and power and cabling concerns. Wireless protocols with source authentication and Advanced Encryption Standard (AES) encryption should be developed to ensure cybersecurity. Channel independence and redundancy techniques should be deployed to ensure failure in one channel which should not cause failure in another. Other methods to preserve channel independence such as geographical separation of the wireless transceivers, independent power sources, minimal inter-channel communication, and high-reliability protocol and software designs should be explored.

\section{Development of Real-Time Multi-Hazard Alert and Damage Assessment Software System}

The above mentioned CNFA sensor system was used to detect hazardous events. However, for civil infrastructures, it is equally important to assess the impact the structure sustained during the hazardous event to evaluate the soundness of the civil infrastructure after the impact. CNFAs are concrete structures, and they experience the same strain during a hazardous event as the civil infrastructure it monitors. We can develop a real-time multihazard alert and damage assessment software system which can record, and aggregate sensor data produced by CNFAs, generate proper alerts when a hazardous event is identified, and allow damage to the civil structures to be assessed after the hazardous 
events. The software can deploy various algorithms for different types of hazards. For example, during a flooding situation, the water level can be detected in real-time using mapping between the ERV and the stress (Figure 3) and between the ERV and the water level (Figure 4) derived at UH. The recorded stress and water level data are also critical for post-flooding assessment as they precisely depict the rising and receding of flood water and the water damage the civil infrastructure experienced during the flooding event. Such data can also be instrumental in evaluating and improving the flood resilience of the civil structural design.

For earthquake detection and damage assessment, the software can track the stress of the CNFAs using both experimental data and models developed. Such data can be used to issue the pre-earthquake warning, earthquake alert as well as post-earthquake infrastructure damage assessment. Research has shown that abnormal seismic activities occur before an earthquake, known as earthquake precursors $[29,30]$. The anomalous amplitude of the strain signal can reach as high as ten times that of ordinary background, and compressive oscillation and extensional oscillation constantly occur before the earthquake [31]. Before the onset of an imminent earthquake, the CNFA sensors can pick up stresses induced by the increasing seismic activities both in its strength and its frequency. The alert system can potentially generate pre-earthquake warnings to ensure that proper damage mitigation measures are taken. During the onset of an earthquake, the system can measure the impact to the civil infrastructure and help post-earthquake damage assessment. Post-earthquake evaluation capability is especially critical for civil structures that do not present any visual damage as it preserves the second-by-second stress level the civil structures experience during the earthquake.

\section{Discussion}

In the preliminary analysis of the ERV measuring circuit, it was noted that the four-probe method of resistance measurement is basically to eliminate the wire and contact resistance while measuring the very low resistance. Since the CNFA aggregates have a very high resistance, using the two-probe method is also applicable. Further, the DC resistance of CNFAs was found to be varying with time, whereas using an $\mathrm{AC}$ circuit to measure the response of stress on CNFAs was found to be more stable. Thus, the use of two wire impedance measurements in the AC circuit can be a future work in the CNFAs advancement.

\section{Conclusion}

Structural health monitoring is an important aspect of the maintenance of large civil infrastructures. Due to the above, mentioned successful development of the piezoelectric response of CNFA in strain, temperature, and hydration monitoring [1] at the University of Houston, this paper presents the use of CNFAs in multi-hazard structural health monitoring of civil infrastructures. The paper also presents the development of a wireless CNFA sensor system which can be radiation-tolerant, have a low noise electrical interface, real-time data acquisition and processing. The system can alert and help to assess impact caused by other natural and human- made disasters such as lightning, airplane approaching, and missile attacks, each of which produces a unique vibration pattern to the CNFA sensors. The multi-hazard alert and damage assessment software system continuously monitors the civil infrastructures, couples the historical data with real-time data for decision making, and provides hazard-specific damage assessment tools for postdisaster structural health analysis.

\section{Acknowledgement}

The research described in this paper is financially supported by the Hurricane Resilience Research

Institute program. The opinions expressed in this study are those of the authors and do not necessarily reflect the views of the sponsor.

\section{References}

1. Howser RN (2013) Development of multifunctional carbon nanofiber aggregate for concrete structural health monitoring. Doctoral dissertation, University of Houston, Houston, USA.

2. Kline CR (2013) Surface-etched etched alumina/SiC mini-whisker composite material and uses thereof. US Patent Number, 8426328 B2.

3. Bontea DM, Chung DDL, Lee GC (2000) Damage in carbon fiber reinforced concrete, monitored by electrical resistance measurement. Cement and Concrete Research 30(4): 651-659.

4. Chen PW, Chung DDL (1996) Concrete as a new strain/stress sensor. Composites Part B: Engineering 27(1): 11-23.

5. Chung DDL (1995) Strain sensors based on the electrical resistance change accompanying the reversible pull-out of conducting short fibers in a less conducting matrix. Smart Materials and Structures 4(1): 59-61.

6. Gao D, Sturm M, Mo YL (2009) Electrical resistance of carbon-nanofiber concrete. Smart Materials and Structures 18(9).

7. Li H, Xiao H, Yuan J, Ou J (2004) Microstructure of cement mortar with nano-particles. Composites Part B: Engineering 35(2): 185-189.

8. Liao WC, Chao SH, Park SY, Naaman AE (2006) Self-consolidating high performance fiber reinforced concrete (SCHPFRC)-preliminary investigation. Report No. UMCEE 06-02, USA.

9. ACI Committee 318. (2014) Building code requirements for structural concrete (ACI 318-14) and commentary. American Concrete Institute. Farmington Hills, MI, USA.

10. Beshay JD, Chen J, Subramania KS, Mahabeleshwara N, Nourbakhsha E, et al. (2016) Wireless networking testbed and emulator (WiNeTestEr). Computer Comminications 73: 99-107.

11. You Y, Chen J, Fengb Y, Tangb Y, Huang D, et al. (2015) A 12GHz low-jitter LC-VCO PLL in 130nm CMOS. Journal of Instrumentation, JINST 10(03): C03013.

12. Xiao L, Chen J (2016) LOCx2 a low-latency, low-overhead 2x5.12gbps transmitter ASIC for the ATLAS liquid argon calorimeter trigger upgrade. Journal of Instrumentation JINST 11(02): C02013.

13. Xiao L, Chen J, Liub C, Liub T, Chen H, et al. (2016) The clock and control system for the ATLAS liquid argon calorimeter phase-I upgrade. Journal of Instrumentation JINST 11(01): C01062.

14. Xiao L, Chen J, Gongb D, Liu T, Fan Q et al. (2017) A low-power low-latency dual-channel serializer ASIC for detector front-end readout. Journal of Instrumentation JINST 12: C01049.

15. Feng Y, Chen J, Youb Y, Tanga Y, Fan Q et al. (2017) A low-power 12.5Gbps serial link transmitter ASIC for particle detectors in $65 \mathrm{~nm}$ CMOS. Journal of Instrumentation JINST 12: C02063. 
16. Guo D, Chen J (2017) Developments of two 4x10Gb/s VCSEL Array Drivers in $65 \mathrm{~nm}$ CMOS for high energy physics experiments. Journal of Instrumentation JINST 12: C02065.

17. You Y, Chen J, Fengb Y, Tangb Y, Huang D, et al. (2015) SET detection and compensation and its application in PLL Design. Journal of Instrumentation JINST 10(05): C05018.

18. Li X, Chen J, Guo D, Hou S, Huang G, et al (2015) 8-Gbps-per-channel radiation-tolerant VCSEL drivers for the HL-LHC detector upgrade. Journal of Instrumentation 10(2): C02017.

19. Deng B, Chen J, Chen H, Chen K, Guo D, et al (2015) The clock distribution system for the ATLAS liquid argon calorimeter phase-I upgrade demonstrator. Journal of Instrumentation 10(1): C01004.

20. Guo D, Chen J, Liu C, Gong D, Hou S, et al (2015) The VCSEL-based array optical transmission (ATx) development towards 120-Gbps link for collider detector: development update. Journal of Instrumentation 10(1): C01034.

21. Deng B, Chen J, Liu C, Gong D, Guo D, et al. (2015) JTAG-based remote configuration of FPGAs over optical fibers. Journal of Instrumentation 10(1): C01050.

22. Deng B, Chen J, HE M, Gong D, Guo D, et al. (2015) Component prototypes towards a low-latency, small-from-factor optical link for the ATLAS liquid argon calorimeter phase-I trigger upgrade. IEEE Transactions on Nuclear Science 62(1): 250-256.

23. Deng B, Chen J (2014) A line-code with quick-resynchronization capacity and low latency for the optical data links of LHC experiments. Journal of Instrumentation 9(7): P07020.
24. Li X, Chen J, Liu G, Deng B, Guo B, et al (2014) Optical data transmission ASICs for the high-luminosity LHC (HL-LHC) experiments. Journal of Instrumentation 9(3): C03007.

25. Guo D, Chen J (2014) The 120Gbps VCSEL array optical transmitter (ATx) development for the high-luminosity LHC (HL-LHC) experiments. Journal of Instrumentation 9(2): C02007.

26. Liang F, Chen J, Lu W, Deng B, Guo D, et al. (2013) A 54-mW 8-Gbps VCSEL Driver in 65-nm CMOS technology. Journal of Instrumentation 9(1): C01021.

27. You Y, Chen J (2014) Radiation-hardened-by-design clocking circuits in 0.13um CMOS technology. Journal of Instrumentation 9(1): C01029.

28. Tang Y, Chen J, Feng Y, Zuo Z, Fan Q, et al. (2016) Wideband LNA with $1.9 \mathrm{~dB}$ Noise figure in $180 \mathrm{~nm}$ CMOS for high-resolution ultrasound imaging applications. IEEE NEWCAS 2016, Vancouver, Canada.

29. Du Q, Sun W, Zeng Z (2016) Stress-strain sensor for monitoring seismic precursors and fault activities in the sand, SPIE smart structures and materials. Non-destructive Evaluation and Health Monitoring, Las Vegas, USA.

30. Mo YL, Howser R, Dhonde H, Song G (2017) Systems and methods utilizing carbon nanofiber aggregate for performance monitoring of concrete structures. US Patent Number: 9797937.

31. Zhang W, Huang W, Li L, Liu W, Li F (2016) High resolution strain sensor for earthquake precursor observation and earthquake monitoring. Sixth European Workshop on Optical Fibre Sensors (EWOFS'2016), Limerick, Ireland
Creative Commons Attribution 4.0 International License

For possible submissions Click Here

\section{Submit Article}

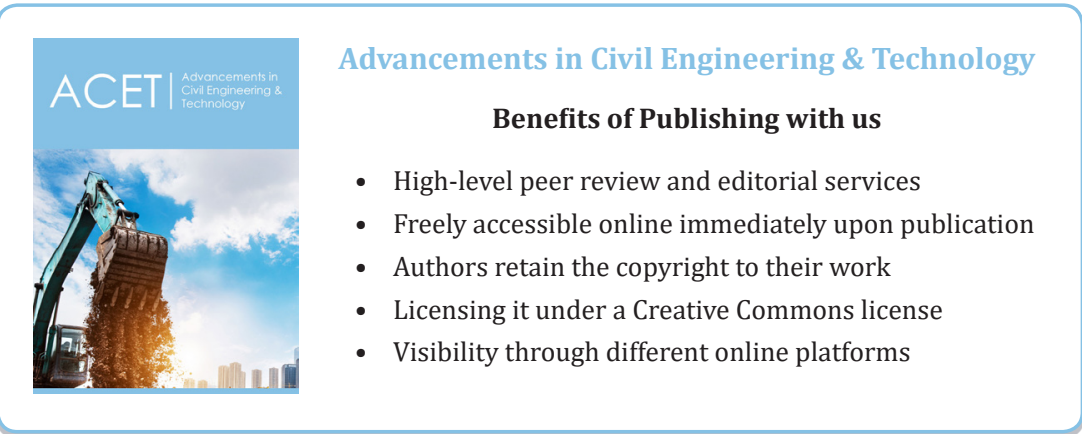

